\title{
RIEMANN $R_{1}$-SUMMABILITY OF INDEPENDENT, IDENTICALLY DISTRIBUTED RANDOM VARIABLES
}

\begin{abstract}
JACK CUZICK
Abstract. Let $X, X_{1}, X_{2}, \ldots$ be i.i.d. random variables. It is shown that $E|X| \log ^{+} \log ^{+}|X|<\infty$ is a sufficient condition for Riemann $R_{1}$-summability of $\left\{X_{n}\right\}$ to $E X$. Counterexamples are provided which indicate that the strongest possible necessary condition of moment type is $E|X|<\infty$. However under weak regularity conditions on the tails of the distribution of $X$ the sufficient condition is also shown to be necessary.
\end{abstract}

1. Introduction. Many regular forms of summability have been studied in great detail for independent, identically distributed random variables $X, X_{1}, X_{2}, \ldots$; the classic example being the strong law of large numbers which asserts that $E|X|<$ $\infty$ iff $\left\{X_{n}\right\}$ is Cesàro $(C, 1)$ summable to $E X$. Lai [5] has shown that the existence of the first absolute moment is also necessary and sufficient for Cesàro $(C, \alpha)$ summability for all $\alpha \geqslant 1$ as well as for Abel summability, whereas Chow [1] in studying Euler $(E, q), q>0$, summability and Borel summability found that the existence of a second moment was equivalent to summability. However, results for Riemann $R_{1}$-summability are not so tidy. Recall that a sequence of real numbers $\left\{A_{n}\right\}$ is $R_{1}$-summable to $A$ if

$$
\lim _{t \rightarrow 0^{+}} \frac{2}{\pi} \sum_{n=1}^{\infty} n^{-1} A_{n} \sin n t=A .
$$

It is well known [3] that this is not a regular summability method. More importantly from our point of view, it is a discrete to continuous method in that the limit in (1) is taken through a continuum of values. In a recent paper Cuzick and Lai [2] have shown that a sequence of i.i.d. random variables $X, X_{1}, X_{2}, \ldots$ is $R_{1}$-summable to $E X$ if $E|X| \log ^{+}|X|<\infty$ and also that $E|X|<\infty$ is a necessary condition for summability. The approach there, as also followed here, is to treat (1) as a random Fourier series and, when $E X=0$, to establish uniform convergence. The purpose of the present note is threefold:

(i) to show that

$$
E|X| \log ^{+} \log ^{+}|X|<\infty
$$

is a sufficient condition for $R_{1}$-summability,

(ii) to give examples which demonstrate that in general the condition $E|X|<\infty$ is the strongest possible necessary condition of moment type, i.e., given a monotone

Received by the editors November 19, 1979 and, in revised form, May 29, 1980.

1980 Mathematics Subject Classification. Primary 60F15, 40G99; Secondary 60G17, 60G50, 42 A24.

Key words and phrases. Riemann summability, random Fourier series, almost sure convergence.

(c) American Mathematical Society $0002-9939 / 81 / 0000-0427 / \$ 02.50$ 
function $g(x)$ such that $\lim _{x \rightarrow \infty} g(x) / x=+\infty$ we can find an $R_{1}$-summable random variable $X$ with $E g(|X|)=+\infty$, and

(iii) to show that under a weak regularity condition on the smoothness of the tails of the distribution function of $X$ the condition (2) is in fact necessary and sufficient.

2. Sufficient conditions. As the constant sequence is $R_{1}$-summable we may take $E X=0$. Then $R_{1}$-summability follows from the continuity of

$$
S(t)=\sum_{n=1}^{\infty} n^{-1} X_{n} \sin n t
$$

which in turn follows from the uniform convergence of the series in (3).

THEOREM 1. Assume $E X=0$ and $E|X| \log ^{+} \log ^{+}<\infty$, where $\log ^{+} x=$ $\max (0, \log x)$. Then the series in (3) is uniformly convergent and $\left\{X_{n}\right\}$ is $R_{1}$-summable.

Proof. The proof is a refinement of the techniques used in [2]. Let $X$ have distribution function $F$ and for $\beta>1$ set

$$
\begin{gathered}
X_{n}^{\prime}=X_{n} I_{\left|X_{n}\right|<n / \log ^{\beta} n}, \quad X_{n}^{\prime \prime}=X_{n} I_{n / \log ^{\beta} n<\left|X_{n}\right|<n}, \\
X_{n}^{\prime \prime \prime}=X_{n} I_{\left|X_{n}\right|>n}
\end{gathered}
$$

and write

$$
\begin{aligned}
S(t)= & \sum_{n=1}^{\infty}\left(X_{n}^{\prime}-E X_{n}^{\prime}\right) n^{-1} \sin n t+\sum_{n=1}^{\infty} E X_{n}^{\prime} n^{-1} \sin n t \\
& +\sum_{n=1}^{\infty} X_{n}^{\prime \prime} n^{-1} \sin n t+\sum_{n=1}^{\infty} X_{n}^{\prime \prime \prime} n^{-1} \sin n t \\
= & S_{1}(t)+S_{2}(t)+S_{3}(t)+S_{4}(t), \quad \text { say. }
\end{aligned}
$$

As $E|X|<\infty$ it follows from the Borel-Cantelli lemma that $S_{4}$ has only a finite number of nonzero terms and hence is uniformly convergent. Uniform convergence of $S_{3}$ follows from the fact that

$$
\begin{gathered}
E \sum_{n=3}^{\infty} n^{-1}\left|X_{n}^{\prime \prime}\right|=\sum_{n=3}^{\infty} n^{-1} \int_{n / \log ^{\beta} n<|x|<n}|x| d F(x) \\
\quad<\text { Const. } \int_{-\infty}^{\infty}\left(\sum_{|x|<n<|x| \log ^{\beta}|x|} n^{-1}\right)|x| d F(x) \\
\quad \text { C Const. } \int_{-\infty}^{\infty}|x| \log ^{+} \log ^{+}|x| d F(x)<\infty
\end{gathered}
$$

The uniform convergence of $S_{1}(t)$ follows from a result of Marcus [7] (see also [2, remarks after Theorem 3]) by checking that

$$
\sum_{n=3}^{\infty}\left[\left\{\sum_{k=n}^{\infty} E\left(X_{k}^{\prime}\right)^{2} k^{-2}\right\}^{1 / 2} / n \log ^{1 / 2} n\right]<\infty
$$


which follows from the estimate

$$
\begin{aligned}
\sum_{k=n}^{\infty} E\left(X_{n}^{\prime}\right)^{2} k^{2}= & \sum_{k=n}^{\infty} k^{-2} \int_{|x|<k / \log ^{\beta} k} x^{2} d F(x) \\
= & \int_{|x|<n / \log ^{\beta} n}\left(\sum_{k=n}^{\infty} k^{-2}\right) x^{2} d F(x) \\
& +\int_{|x|>n / \log ^{\beta} n}\left(\sum_{k / \log ^{\beta} k>|x|} k^{-2}\right) x^{2} d F(x) \\
= & O(\log n)^{-\beta} \quad \text { since } \beta>1 .
\end{aligned}
$$

As $E X=0$, we may replace $E X_{n}^{\prime}$ by $E X I_{|X|>n / \log _{n}{ }_{n}}$ in $S_{2}$. Interchanging summation and expectation as before we find the $N$ th partial sum of $S_{2}(t)$ equals

$$
\int_{-\infty}^{\infty}\left(\sum_{n / \log ^{\beta} n<x}^{N} n^{-1} \sin n t\right) x d F(x) .
$$

If we compute

$$
C(i, t)=\sup _{j}\left|\sum_{i<n<j} n^{-1} \sin n t\right|
$$

and

$$
C=\sup _{i, t} C(i, t)
$$

it is easy to check that $C<\infty$ and $C(i, t) \downarrow 0$ as $i \uparrow \infty$ uniformly for $t$ on compact sets not containing $\{2 \pi n\}_{n=-\infty}^{\infty}$. From this it follows that $S_{2}(t)$ is uniformly convergent on such sets and we need only concern ourselves with uniform convergence in a neighborhood of the origin, i.e. given $\varepsilon>0$, it is enough to find a $\delta>0$ such that when $|t|<\delta$, (4) is less than $\varepsilon$ for all $N$. Choose $D$ so that

$$
\int_{|x|>D}|x| d F(x)<\varepsilon / 2 C
$$

Then the integral (4) on the set $|x|>D$ is less than $\varepsilon / 2$ and it suffices to show that for all $N$

$$
\int_{|x|<D}\left(\sum_{N<n / \log ^{\beta} n<x} n^{-1} \sin n t\right) x d F(x)<\varepsilon / 2
$$

for all $t$ sufficiently small. This follows immediately from the convergence to zero of the integrand as $t \rightarrow 0$ uniformly for $|x|<D$, as the series contains only a finite number of terms for $|x|$ bounded.

3. Necessary conditions. Cuzick and Lai [2] noted that $E|X|<\infty$ was necessary for $R_{1}$-convergence. The following example indicates that no stronger necessary condition of moment type can be found. Let $g(x)$ be any function such that $g(x) / x \uparrow \infty$ as $x \rightarrow \infty$. Choose any $0<\varepsilon<1$ and let $n_{k}$ be the first $n>2 n_{k-1}$ and of the form $2^{j} \mathrm{n}$ for some $j>1$ such that $g\left(n_{k}\right) / n_{k}>k^{2+e}$. Let $X$ have mass $M /\left(n_{k} k^{2+e}\right)$ at $\pm n_{k}$ with $M$ chosen so that the total mass is unity. Then $E|X|<\infty$ but $E g(|X|)=+\infty$. As $E\left|X_{n}\right|<\infty$, by the Borel-Cantelli lemma we may replace 
$X_{n}$ by $X_{n}^{\prime}=X_{n} I_{\left|X_{n}\right|<n}$ and change only a finite number of terms. Now for $n_{k}<2^{n}$ $<n_{k+1}$ compute

$$
s_{n}=\left(\sum_{j=2^{n}+1}^{2^{n+1}} E\left(X_{j}^{\prime} / j\right)^{2}\right)^{1 / 2}=O\left(\frac{n_{k}}{2^{n} k^{2+e}}\right)^{1 / 2} .
$$

Then we may choose $s_{n}^{*}>s_{n}$, with $s_{n}^{*}$ nonincreasing so that

$$
\sum_{n=1}^{\infty} s_{n}^{*}=\sum_{k=1}^{\infty}\left(\sum_{n_{k}<2^{n}<n_{k+1}} s_{n}^{*}\right)=O\left(\sum_{k=1}^{\infty} k^{-(1+e / 2)}\right)<\infty .
$$

It follows from a result of Kahane [4, p. 65, Remark 2] that

$$
\sum_{n=1}^{\infty} X_{n}^{\prime} n^{-1} \sin n t
$$

is uniformly convergent and thus $\left\{X_{n}\right\}$ is $R_{1}$-summable.

This result relies heavily on the lacunary behavior of the distribution of $X$. If some mild conditions are placed on the regularity of the tails of the distribution of $X$, it is possible to show that (2) is also necessary for $R_{1}$-summability.

THEOREM 2. Assume that for all $n$ sufficiently large

$$
\int_{2^{n}<|x|<2^{n+1}}|x| d F(x) \text { is nonincreasing as } n \uparrow \infty \text {. }
$$

Then $\left\{X_{n}\right\}$ is Riemann $R_{1}$-summable $\Leftrightarrow E|X| \log ^{+} \log ^{+}|X|<\infty$.

Proof. We need only establish that under (5), condition (2) is necessary for summability. First assume $X$ is symmetric. We shall need the results of $\S 3$ in [2]. Set $W_{n}=X I_{0<X<n}$ and let $G_{n}$ be the unique solution of the equation

$$
n E\left(\min \left\{\left|W_{n} / G_{n}\right|,\left(W_{n} / G_{n}\right)^{2}\right\}\right)=1
$$

If $\left\{X_{n}\right\}$ is summable it follows from Theorem 5 of [2] that

$$
\sum_{n=1}^{\infty} \gamma_{n}<\infty
$$

where $\gamma_{n}=G_{2^{n}} / 2^{n}$. We aim to show that

$$
\gamma_{n}=o\left(\frac{1}{n}\right)
$$

which follows from (7) if we can establish that $\gamma_{n}$ is "almost monotone" in the sense that there exists an $n_{0}$ and $K_{1}>0$ such that

$$
\gamma_{n}>K_{1} \gamma_{m} \text { for all } m>n>n_{0} \text {. }
$$

To prove this we need to verify the existence of an $n_{0}$ and $K_{2}>0$ such that for all $n>j \geqslant n_{0}$

$$
2^{-n} \int_{0}^{2^{n}} x^{2} d F(x) \geqslant K_{2} 2^{-(n+k)} \int_{0}^{2^{n+k}} x^{2} d F(x), \text { all } k>0
$$

and

$$
\int_{2^{j}}^{2^{n}} x d F(x)>K_{2} \int_{2^{j+k}}^{2^{n+k}} x d F(x), \text { all } k>0
$$


Equation (11) follows immediately from (5) and (10) follows from (5) upon noting that

$$
\begin{aligned}
\int_{2^{n_{0}+1}}^{2^{n}} x^{2} d F(x) & =\sum_{m=n_{0}}^{n} \int_{2^{m-1}}^{2^{m}} x^{2} d F(x) \\
& \geqslant \sum_{m=n_{0}}^{n} 2^{m-1} \int_{2^{m-1}}^{2^{m}} x d F(x) \geqslant \sum_{m=n_{0}}^{n} 2^{m-1} \int_{2^{m+k-1}}^{2^{m+k}} x d F(x) \\
& \geqslant \sum_{m=n_{0}}^{n} 2^{k-2} \int_{2^{m+k-1}}^{2^{m+k}} x^{2} d F(x) \geqslant \frac{1}{4} 2^{-k} \int_{2^{n_{0}+1}}^{2^{n+k}} x^{2} d F(x) .
\end{aligned}
$$

Now for $0<G \leqslant 2^{n}$, define

$$
g_{n}(G)=\int_{0}^{G} \frac{x^{2}}{G} d F(x)+\int_{G}^{2^{n}} x d F(x)
$$

Then $g_{n}$ is nonincreasing in $G$. Choose $\beta_{n}$ of the form $2^{i}$ for some integer $i$ such that $\frac{1}{2} \beta_{n}<G_{2^{n}}<\beta_{n}$. Then using (6)

$$
\gamma_{n}=g_{n}\left(G_{2^{n}}\right) \geqslant g_{n}\left(\beta_{n}\right)
$$

which from (10) and (11) is greater than or equal to $K_{2} g_{n+k}\left(2^{k} \beta_{n}\right)$ for all $k>0$. If $2^{k} \beta_{n}<G_{2^{n+k}}$ then

$$
K_{2} g_{n+k}\left(2^{k} \beta_{n}\right) \geqslant K_{2} g_{n+k}\left(G_{2^{n+k}}\right)=K_{2} \gamma_{n+k}
$$

so that (9) holds. Of course (9) also holds when $2^{k} \beta_{n}>G_{2^{n+k}}$ since then $G_{2^{n}}>\frac{1}{2} \beta_{n}$ $>2^{-(k+1)} G_{2^{n+k}}$ so that $\gamma_{n}=G_{2^{n}} / 2^{n} \geqslant \frac{1}{2} \gamma_{n+k}$.

Now define $G(x)=G_{2^{n}}$ for $x=n$ and by linear interpolation for nonintegral $x$. It is easily checked that $G$ is nondecreasing. From (8) it follows that there exists $K_{3}>0$, such that for sufficiently large $x$

$$
G^{-1}(x) \geqslant \log x+K_{3} \log \log x .
$$

It follows from (7), (13) and (12) that

$$
\sum_{n=1}^{\infty} \int_{G(n)}^{2^{n}} x d F(x)<\infty
$$

and a Fubini argument shows

$$
\int^{\infty}\left[G^{-1}(x)-\log x\right] x d F(x)<\infty
$$

from whence (2) follows with the help of (14). This establishes the theorem when $X$ is symmetric. In general let $m$ be a median of $X$ and for $2^{k}<n<2^{k+1}$ define

$$
Y_{n}=\left(X_{n}-m\right) I_{\left|X_{n}-m\right|<2^{k-1}}
$$

and let $Z_{n}$ be the symmetrized version of $Y_{n}$, i.e. $Z_{n}=Y_{n}-Y_{n}^{\prime}$ where $Y_{n}, Y_{n}^{\prime}$ are i.i.d. Then $\left\{X_{n}\right\}$-summable $\Rightarrow\left\{Z_{n}\right\}$-summable

$$
\Rightarrow \sum_{k=1}^{\infty} \gamma_{k}^{Z}<\infty \text { as at (7) }
$$

where $\gamma_{k}^{Z}=G_{2^{k}}^{Z} / 2^{k}$ and $G_{2^{k}}^{Z}$ is the unique solution of (6) with $W_{n}$ replaced by $Z_{2^{k}}$. 
Define $\gamma_{k}^{Y}$ and $G_{2^{k}}^{Y}$ similarly. Then integration by parts and the weak symmetrization inequality $[6$, p. 245] gives

$$
\begin{aligned}
\gamma_{k}^{Z} & =E\left(\min \left\{Z_{2^{k}}^{2} / G_{2^{k}},\left|Z_{2^{k}}\right|\right\}\right)=\int_{0}^{\infty} \min \left\{x^{2} / G_{2^{k}}^{Z}, x\right\} d F_{\left|Z_{2^{k}}\right|}(x) \\
& \geqslant \frac{1}{2} \int_{0}^{\infty}\left(1-F_{\left|Z_{2^{k}}\right|}(x)\right) \min \left\{2 x / G_{2^{k}}^{Z}, 2\right\} d x \\
& \geqslant \frac{1}{4} \int_{0}^{\infty}\left(1-F_{\left|Y_{2^{k}}\right|}\left(\frac{x}{2}\right)\right) \min \left\{2 x / G_{2^{k}}^{Z}, 2\right\} d x \\
& \geqslant \frac{1}{16} \int_{0}^{\infty}\left(1-F_{\left|Y_{2^{k}}\right|}(x)\right) \min \left\{2 x / G_{2^{k}}^{Z}, 2\right\} d x \\
& \geqslant \frac{1}{16} E\left(\min \left\{Y_{2^{k}}^{2} / G_{2^{k}}^{Z},\left|Y_{2^{k}}\right|\right\}\right) .
\end{aligned}
$$

From this it can be shown as in the argument following (13) that

$$
\gamma_{k}^{Z} \geqslant \frac{1}{8} \gamma_{k}^{Y} \text {. }
$$

It is easily verified from (6) that for $k$ large there exists $K_{3}>0$ such that

$$
G_{2^{k}}^{Y}>K_{3} 2^{k / 2} \text {. }
$$

Now for $G_{2^{k}}^{Y}>2 m$ we have

$$
\begin{aligned}
\gamma_{k}^{Y} & =E\left(\min \left\{Y_{2^{k}}^{2} / G_{2^{k}}^{Y},\left|Y_{n}\right|\right\}\right) \\
& =E\left(\min \left\{(X-m)^{2} I_{|X-m|<2^{k-1}} / G_{2^{k}}^{Y},|X-m| I_{|X-m|<2^{k-1}}\right\}\right) \\
& \geqslant \frac{1}{2} E\left(\min \left\{X^{2} I_{|X|<2^{k}} / G_{2^{k}}^{Y},|X| I_{|X|<2^{k}}\right\}-m^{2} / G_{2^{k}}^{Y}\right) \\
& \geqslant \frac{1}{2}\left(\gamma_{k}^{X}-m^{2} / G_{2^{k}}^{Y}\right) .
\end{aligned}
$$

Combining (15), (16) and (17) gives

$$
\sum_{k=1}^{\infty} \gamma_{k}^{X}<\infty
$$

If we let $|V| \stackrel{D}{=}|X|$ and let $V$ be symmetric, i.e. $V= \pm X$, then $\gamma_{k}^{V}=\gamma_{k}^{X}$ and, as $V$ satisfies (5) and is symmetric, we may use the proof previously given to see that (18) implies $\left\{V_{n}\right\}$ is summable

$$
\Rightarrow E|X| \log ^{+} \log ^{+}|X|=E|V| \log ^{+} \log ^{+}|V|<\infty
$$

which completes the proof in general.

\section{REFERENCES}

1. Y. S. Chow, Delayed sums and Borel summability of independent, identically distributed random variables, Bull. Inst. Math. Acad. Sinica 1 (1973), 207-220.

2. Jack Cuzick and T. L. Lai, On random Fourier series, Trans. Amer. Math. Soc. 261 (1980), 53-80.

3. G. H. Hardy and W. W. Rogosinski, Notes on Fourier series (V): Summability ( $\left.R_{1}\right)$, Proc. Cambridge Philos. Soc. 45 (1949), 173-185.

4. J. P. Kahane, Some random series of functions, Heath, Lexington, Mass., 1968.

5. T. L. Lai, Summability methods for independent, identically distributed random variables, Proc. Amer. Math. Soc. 45 (1974), 253-261.

6. M. Loeve, Probability theory, 3rd ed., Van Nostrand, Princeton, N. J., 1963.

7. M. B. Marcus, Continuity and the central limit theorem for random trigonemetric series, $\mathrm{Z}$. Wahr. Verw. Gebiete 42 (1978), 35-56.

Mathematical Institute, 24-28 St. GIles, OXford OX1 3LB, England 